\title{
Espaces numériques oppositionnels et mobilisation en Italie
}

Digital oppositional spaces and mobilisation in Italy

\section{Paola Sedda}

\section{(2) OpenEdition \\ 12 Journals}

Édition électronique

URL : http://journals.openedition.org/ctd/1758

DOI : $10.4000 /$ ctd. 1758

ISSN : 2491-1437

Éditeur

Chaire Unesco Pratiques émergentes en technologies et communication pour le développement

Édition imprimée

Date de publication : 12 novembre 2015

\section{Référence électronique}

Paola Sedda, «Espaces numériques oppositionnels et mobilisation en Italie », Communication, technologies et développement [En ligne], 2 | 2015, mis en ligne le 11 juin 2019, consulté le 22 juillet 2020. URL : http://journals.openedition.org/ctd/1758 ; DOI : https://doi.org/10.4000/ctd.1758

Ce document a été généré automatiquement le 22 juillet 2020.

Communication, technologies et développement 


\title{
Espaces numériques oppositionnels et mobilisation en Italie
}

\author{
Digital oppositional spaces and mobilisation in Italy
}

\author{
Paola Sedda
}

\section{Introduction}

1 La démocratisation progressive des usages d'Internet s'est traduite par une effervescence croissante de l'activisme en ligne. En constituant une alternative aux lieux traditionnellement voués au combat social, l'espace public numérique est de la sorte devenu un canal de mobilisation puissant ainsi qu'un terrain propice à la formation et à la propagation de nouveaux cadres contestataires.

2 En refusant d'emblée une posture déterministe, notre travail considère que l'intensification des usages du numérique au sein des nouveaux collectifs contestataires (Cardon, 2013; George, 2008; Granjon, 2001) s'inscrit dans des processus socio-politiques plus larges ayant contribué au détachement de l'action citoyenne des canaux institutionnels (Rosanvallon, 2006; Mathieu, 2011) et à l'émergence de nouvelles formes de militantisme distancié (Ion, Franguiadakis et Viot, 2005). Les chercheurs en Sciences de l'information et de la communication se sont alors promptement penchés sur cette problématique afin d'interroger le rôle joué par Internet dans le développement de nouvelles formes expressives de la participation démocratique (Monnoyer-Smith, 2011), dans l'activation de nouvelles modalités de prise de parole dans l'espace public (Dahlgren, 2009 ; Lecomte, 2009; Cardon 2010; Miège, 2010) ainsi que dans l'évolution des pratiques militantes (Granjon, 2001 ; Flichy, 2008 ; George, 2003).

Mais si les nouvelles dynamiques de médiatisation de l'action collective ont donné lieu à une riche littérature qui ne cesse de s'accroitre, peu d'attention a été consacrée aux discours militants et aux nouveaux processus de formation des cadres protestataires.

En couvrant cet axe de recherche, notre démarche vise ainsi à considérer le réseau Internet non pas uniquement comme un moyen de diffusion et de coordination de la 
lutte mais avant tout comme un nouveau terrain pour la construction discursive de la critique sociale.

\section{Espaces numériques oppositionnels et nouveaux cadres contestataires}

\subsection{Théorie critique, pratiques de communication et action collective}

5 Une perspective axée sur les mécanismes de construction des cadres contestataires nous invite à prendre en considération l'ensemble des occasions d'échange symbolique au sein des nouveaux collectifs ainsi que la manière dont les pratiques numériques contribuent au processus de cadrage de la contestation. La faible attention qui semble avoir été adressée aux discours de l'action collective de la part des approches communicationnelles de la mobilisation reflète la position dominante occupée par le paradigme de la mobilisation des ressources face aux approches cognitivistes. La perspective cognitiviste s'inscrit dans un processus de renouvellement des études sur les mouvements sociaux qui s'amorce au début des années 1980. C'est à partir de cette période que certains sociologues de la mobilisation ont commencé à manifester leur intérêt vers les dimensions culturelles et discursives de l'expérience contestataire afin de contre-balancer la primauté de la perspective stratégique et organisationnelle qui s'était affirmée dans la discipline.

6 Selon l'approche des cadres (frame perspective), le processus de construction discursive $\mathrm{du}$ cadre contestataire, permettant aux individus d'adopter le point de vue du mouvement, constitue un moment crucial pour le passage à l'action contestataire (Snow, 2001). Les cadres contestataires d'un mouvement sont sont donc produits à travers les interactions discursives des membres de l'organisation. Afin qu'ils puissent $\hat{e}$ tre percutants pour les adhérents potentiels, les cadres de la contestation doivent rentrer en résonance avec les valeurs et les croyances enracinées dans la société civile.

7 C'est en vertu de cette cohérence, que le cadre dominant ( master frame ${ }^{1}$ ») choisi par l'organisation, acquiert un potentiel de mobilisation capable d'amener à l'engagement effectif (Snow et Benford, 1992). En soulignant un lien étroit entre les valeurs culturelles et sociales et les représentations promues au sein des mouvements contestataires, cette approche renoue aussi le débat autour de l'idéologie. De nombreuses études montrent en effet une correspondance entre les contenus idé ologiques et le processus de construction des cadres ${ }^{2}$.

8 Les mouvements sociaux, en tant que lieux de production de valeurs et de visions partagées, sont censés activer une vision critique de la culture dominante qui est vé hiculée à travers l'ensemble des appareils idéologiques d'une société3. Le militantisme, en tant que processus collectif de création symbolique visant à modifier un aspect de l'ordre social, est donc toujours porteur d'une démarche idéologique même quand celle-ci n'est pas identifiable avec les clivages politiques stricto sensu. Ce déplacement des idéologies du domaine politique orthodoxe aux autres spectres de la vie sociale n'implique donc pas forcement une absence de la dimension idéologique mais il té moigne plutôt d'un processus de "dilution » du champ politique ayant des impacts variables sur les mécanismes de formation des cadres contestataires. 
9 En Italie, la naissance du Mouvement 5 étoiles a stimulé une riche réflexion autour de l'émergence des nouveaux populismes ainsi que du rôle joué par les nouveaux médias dans la construction des discours sur les modèles de démocratie (Biancalana, 2014). En axant notre analyse sur le processus de cadrage, nous avons toutefois décidé d'éviter le recours systématique à une catégorie sémantique complexe et fourre-tout comme celle de "populisme". . Certainement apte à relever les paradoxes du mouvement en ce qui concerne sa vision et sa pratique de la démocratie digitale (Biancalana, 2014), cette approche aurait pu en effet encourager l'adoption d'une perspective oblitérée. Notre prise de distance n'implique pas toutefois l'absence de convergences avec les travaux italiens.

Bien que beaucoup plus axés sur l'analyse du vote et des appartenances politiques des électeurs du Mouvement 5 étoiles, ceux-ci montrent bien la complexité du phénomène Grillo précisément à partir de la difficulté de le classer à l'intérieur des clivages politiques traditionnels (Gualmini et Corbetta, 2013).

11 L'approche cognitiviste nous a donc permis de travailler plus aisément sur les idéologies, les mythes et les représentations qui ont contribué à l'adoption de la vision critique du blog de la part des activistes-usagers. Dans le champ contestataire, les potentialités liés aux dispositifs d'auto-publication et d'expression de la parole profane semblent avoir encouragé la formation d'espaces info-communicationnels oppositionnels répondant à ses propres formes de communication et à des pratiques de participation et de délibération originales (Negt, 2007, p. 8). Les TIC revêtent en donc ici une fonction idéologisante ayant un impact tant dans la construction des nouveaux cadres collectifs de l'injustice sociale.

12 En faisant dialoguer le concept d'"espace public oppositionnel" d'Oskar Negt avec l'approche des cadres contestataires (Snow et Benford, 1992; 2000) et celle des usages militants d'Internet (Granjon, 2001; George, 2003), nous allons appréhender l'espace numérique non pas en tant que nouvelle architecture de l'action collective (Castells, 2009) mais plutôt comme un espace info-communicationnel conflictuel où les formes de la domination symbolique se heurtent à des structures communicationnelles résistantes (Habermas, 1987).

\subsection{La dialectique de l'espace public}

Bien que pas forcement partisanes ou visant un projet de société défini, les nouvelles modalités de publicisation de la parole citoyenne (via les blogs ou les réseaux sociaux) semblent pouvoir représenter un défi pour l'espace public dominant. Dans cette perspective, l'espace public habermassien n'a peut-être jamais constitué une sphère complètement autonome mais il a toujours hébergé la dialectique interne à toute société : celle entre l'ossature culturelle et sociale dominante et le refus de ses dérives.

14 L'espace public oppositionnel, en accueillant en son sein le potentiel rebelle et imaginatif des couches laborieuses et des groupes marginaux, a précisément la fonction de revitaliser cette dialectique.

15 Selon Oskar Negt, l'impossibilité, pour l'espace public habermassien, "de produire une synthèse réelle des expériences sociales" est due à l'écartement des segments de la soci été, comme la sphère privée et celle du travail, qui sont au fondement de ces mêmes expériences (Negt, 2007, p. 10). Le concept de sphère publique plébéienne développé par Oskar Negt nous permet d'observer empiriquement les phénomènes de prise de 
parole de la part d'acteurs qui ne sont pas reconnus comme légitimes dans l'espace public bourgeois.

En ce sens, l'espace public dominant revêt une fonction conservatrice du mode de production bourgeois plutôt que réformatrice. Pour le dire avec les mots du philosophe francfortois, "il s'agit ici d'un enchevêtrement de normes, de légitimations, de délimitations, de procédures, de séparations du pouvoir, qui empêche l'espace public politique de conduire à des décisions pouvant perturber ou abolir le mode de production bourgeois" (Negt, 2007, p. 95). Il y aurait donc des barrières à la formation d'un espace public politique à proprement dit, capable de modifier radicalement l'ordre social.

('Oskar Negt encourage alors une nouvelle acception plurielle critique de l'espace public habermassien qui peut être appréhendé comme le point où convergent et s'exercent conjointement les forces de légitimation et de dé-légitimation du système.

18 L'espace public politique constitue donc en soi un objet de lutte dans le sens où les mouvements sociaux sont constamment engagés dans la redéfinition de ses frontières, de ses modes d'accès et d'expression. Le penseur critique observe que notre société est caractérisée par un processus de perte des valeurs traditionnelles, d'affaiblissement des liens sociaux et de la dimension publique auxquels correspond la croissance exponentielle du pouvoir du marché.

Ces facteurs ont déterminé l'effondrement de la couche moyenne et l'appauvrissement des dimensions idéologiques et culturelles indispensables à une gestion sociale de l'é conomie (Miège, 2010). "L'espace public oppositionnel ", voué à recueillir et à sé dimenter les expériences oppositionnelles, devrait donner lieu à une délibération permanente au sein des différentes sphères de la société (Negt, 2007).

20 Nettement séparés des partis politiques traditionnels, les nouveaux mouvements sociaux semblent incarner ou, tout du moins, se rapprocher de l'idée d'espace public prolétarien formulée par Oskar Negt. Ces nouveaux espaces se présentent alors comme une variante du modèle bourgeois ayant la fonction de produire une dialectique en son interne ${ }^{5}$.

21 En Italie, c'est dans un contexte de forte défiance citoyenne à l'égard de la politique traditionnelle que maintes initiatives ont vu le jour au cours des dernières années.

Parmi ces nouveaux collectifs ${ }^{6}$, émerge le Mouvement 5 étoiles, l'organisation politique liée au blog du comique Beppe Grillo. Né en 2009 avec l'objectif de stimuler la participation politique par le bas, ce mouvement a réussi à faire converger le désenchantement d'une large partie de citoyens vers la création d'un nouveau sujet politique qui a élu la sphère d'Internet comme principal lieu de construction du discours contestataire et d'élaboration de l'action collective.

Suite au succès électoral remporté en occasion des élections législatives de février $2013^{7}$, le mouvement de Grillo s'est imposé dans l'échiquier politique national en générant une situation de forte instabilité politique dans le pays8. En hébergeant les col ères des précaires, des étudiants et des jeunes professionnels (Corbetta et Gualmini, 2013), le blog de Beppe Grillo a démontré sa capacité à fédérer les expériences vécues et à canaliser les problèmes substantiels de la société italienne. Comme il a été suggéré par Oskar Negt, ceux-ci n'apparaissent « que de façon superficielle dans l'espace public bourgeois tel qu'il se manifeste » (Negt, 2007). 


\section{L'Internet contestataire italien : le cas du Mouvement 5 étoiles}

\subsection{La mobilisation vue par le web : méthodologie et outils d'analyse}

Notre enquête a été construite autour des méthodes de l'observation participante et de l'analyse du contenu. En s'éloignant de l'idéal-type de l'espace public habermassien, le blog fait converger une multitude de registres, de formats et de formes énonciatives. Les commentaires, les témoignages, les photo-montages et les posts contribuent à alimenter une critique radicale de la politique nationale et européenne. Ainsi, l'adoption du concept d'espace public oppositionnel nous a également permis de définir plus clairement notre terrain et de l'approcher d'une manière empirique.

25 À partir d'un cadre conceptuel fortement ancré dans la Théorie critique, nous avons alors essayé d'identifier le rôle du blog dans le processus de construction et d'appropriation du cadre contestataire et de comprendre l'importance revêtue par les activités info-communicationnelles dans les dynamiques de la mobilisation et de la participation politique.

Nous avons alors effectué une immersion dans la communauté en en devenant un membre à tous les effets (inscription sur la plateforme, abonnement à la newsletter et aux profils créés dans les différents réseaux sociaux, participation épisodique au débat). La longue période d'observation (presque quatre années consécutives, de 2009 à 2013) a alors consisté dans la consultation quotidienne des posts principaux et des commentaires rédigés par les internautes.

Sur des portions isolées de texte, nous avons ensuite mené une analyse de contenu approfondie afin d'identifier les thèmes abordés dans le blog et les idéaux mobilisés.

Nous avons aussi considéré l'importance d'analyser la structure du blog, l'organisation du contenu et les fonctionnalités à destination des internautes. L'analyse d'un corpus numérique défini en amont et composé par les 53 communiqués politiques de Beppe Grillo, par le «non-statut » et le programme politique du mouvement, a donc été associée à l'analyse structurelle de l'espace du blog. Puisque nous avons privilégié une approche qualitative, l'analyse de contenu a été effectuée manuellement. Nous avons en effet effectué une sélection de posts politiquement très significatifs et étalés dans le temps (des premiers balbutiements de la communauté des grillini jusqu'à la naissance du mouvement). Nous avons alors créé des tableaux sur lesquels nous avons catalogué les expressions saillantes du discours politique en les organisant à la fois à partir du domaine sémantique et de la logique de la polarisation présente dans le blog. Ce choix nous a permis également d'adopter une perspective diachronique, indispensable pour relever le processus de construction discursive de la critique sociale militante.

\subsection{L'analyse du blog : structure, contenu et fonctionnement}

Le discours contestataire du Mouvement 5 étoiles a été construit sur le blog de contreinformation politique de Beppe Grillo. Ce dernier constitue le principal outil communicationnel du réseau des militants et le lieu où convergent toutes les activités liées au mouvement. L'analyse de la structure et du contenu du blog a contribué à faire 
émerger la nature complexe de cet espace qui fonctionne à la fois comme plateforme d'information et d'échange et comme moyen de mobilisation et d'action politique. personnels. En ce sens, la perspective anti-hégémonique et celle expressiviste (Cardon et Granjon, 2010) apparaissent comme indissociables et contribuent mutuellement à la construction d'une nouvelle politique de l'émancipation. Le développement des pratiques info-communicationnelles militantes à l'intérieur du blog correspond aussi à une nouvelle approche du débat politique qui, dans la logique du mouvement, doit se fonder sur la libre confrontation des idées et non sur des idéologies acceptées a priori. Par le biais d'une parole politique bruyante et diffuse, l'espace du blog rend donc manifeste l'interdépendance entre la sphère privée et l'espace public, celle-ci étant constitutive du langage, des formes de distribution ainsi que de la sphère publique ellemême (Negt, p. 57). Le statut hybride du blog, fonctionnant à la fois comme réceptacle du mécontentement et comme outil d'action politique, nous permet donc d'identifier une tendance générale vers la désacralisation et la dé-professionnalisation de la politique.

\subsection{Le communiqué politique comme outil de cadrage}

La structure du blog permet de distinguer l'espace dédié à la discussion autour de l'actualité politique (espace central de socialisation et de fidélisation des militants) de 
l'espace dédié à la communication et à l'action militante (la section dédiée au Mouvement 5 étoiles). partis sont un mur qui tous les jours a une nouvelle fissure "; « aucun parti ne pourra gouverner longtemps sur les décombres qu'il a créé», «la vieille politique est en déclin ", "les partis sont morts ». Les expressions qui renvoient au sens du néant sont également très nombreuses : " tout change pour que rien ne change ", « ils ne discutent de rien ", « ils ne sont rien », « le mot politicien ne veut plus rien dire ». Les partis sont considérés comme un corps étranger dans la démocratie ( $«$ un corps séparé de la société »), comme une force occupante (« ils occupent les institutions»). Les discours sont donc polarisés à travers une opposition constante entre les attributs du vieux monde et ceux du nouveau monde, entre l'état de domination et de "servitude ${ }^{12} \mathrm{du}$ passé et les promesses de participation du futur. positif a la fonction de véhiculer une rhétorique du changement. L'action du mouvement est alors associée à des expressions telles que "changement d'époque ", " contagion ", « tremblement social ", « fait révolutionnaire ", « renversement ».

L'entrée du Mouvement 5 étoiles dans la politique nationale est donc conçue et vécue comme une révolution, à la fois politique et sociale. Cette révolution s'opère avant tout par le biais de " la participation directe des italiens à la chose publique ». La " démocratie par le bas ", constituant le premier mot proprement politique prononcé par le leader dans ses communiqués, constitue donc le macro-cadre du mouvement (master frame).

Bien que réprimé ou désavoué au cours de l'histoire, de la Révolution française à la Commune jusqu'à l'élan participatif qui a animé mai '68, l'idéal de la démocratie directe 
constitue un cadre contestataire historique et toujours actuel. Cet idéal de démocratie, directe, participative, venant du bas, se situe dans la sphère sémantique de la vie et du futur. « Nous sommes vivants ", « nous sommes les fous de la démocratie »; « au sein du Mouvement 5 étoiles, la démocratie directe est une réalité quotidienne ». Le Mouvement 5 étoiles signerait alors l'avènement d'une « démocratie populaire qui prétend décider et contrôler le destin de son pays, de sa cité, de sa vie».

$\mathrm{Si}$, à première vue, la centralité de concepts tels que souveraineté, participation ou démocratie populaires semble suggérer une stratégie discursive empruntée essentiellement au populisme, en réalité, le nombre d'occurrences du mot «citoyen » dépasse largement celui des mots renvoyant au «peuple».

À la place d'une référence abstraite à la volonté populaire et à la spontanéité de ses aspirations, les discours du mouvement se focalisent sur l'individu. L'emphase est alors mise sur l'idéal moderne de l'autonomie, sur le réveil des consciences, sur la responsabilité individuelle ${ }^{13}$. C'est en cela que la philosophie libertaire assume une place de premier plan dans la construction du cadre contestataire du collectif en véhiculant une dimension idéologique forte qui est intimement liée à l'utopie d'Internet.

Dans les discours du mouvement, l'idée de « la fin de la politique » (Mosco, 2004) ${ }^{14}$ est indissociable de la pratique émancipatrice du réseau. Le réseau est défini dans le blog comme "le nouveau Spartacus ", capable d'offrir les moyens pour se révolter contre l'esclavage. Grillo affirme clairement que « le réseau produit la somme des intelligences des citoyens et que celle-ci possède une valeur supérieure à n'importe quel politicien de profession». Le réseau, en tant que système horizontal basé sur l'interconnexion généralisée et le dynamisme (Musso, 2003), s'oppose à la structure verticale et pyramidale du parti. Il est en effet défini comme le vecteur de la délibération collective (" le programme a été élaboré en réseau ») et associé à la transparence dans la gestion de la chose publique («le réseau est le gardien du processus décisionnel»). Le réseau correspond aussi au lieu du changement par excellence («le Mouvement 5 étoiles est un mouvement open source, sans adresses, assemblées ni modes de coordination»). L'absence d'idéologies est donc seulement apparente car le mouvement manifeste une foi presque religieuse dans le potentiel créateur et transformateur d'Internet.

Les discours politiques sont toutefois accompagnés par une variété de symboles issus de la culture populaire et télévisuelle et utilisés d'une manière syncrétique. En refusant les idéologies politiques orthodoxes, le mouvement acquiert une grande liberté dans la réappropriation de discours, traditions et valeurs, historiquement ou culturellement très éloignés. Le blog mélange ainsi les temporalités, les événements, les styles, en produisant l'idée d'un présent permanent qui demeure par contre toujours insaisissable et changeant (Bauman, 2013; Rosa, 2012). Il est donc parfois très difficile de distinguer la logique strictement politique et émancipatrice des logiques empruntées au spectacle, au divertissement et à la "marketisation" du message (Bauman, 2013). Ainsi, si la satire transporte en soi la critique, elle semble avoir aussi une autre fonction dans le blog: celle de captiver l'usager, de le faire adhérer à un univers symbolique où le sens de la révolte et de l'engagement semble parfois incompatible avec son rire distrait. 


\subsection{Le discours politique du mouvement et ses paradoxes}

\section{1. présentée ci-bas) est à la base du processus discursif de construction du cadre} contestataire. Le cadre contestataire est donc produit à partir du diagnostic critique qui est effectué sur la politique officielle. L'analyse du blog nous a ainsi conduit à identifier un ensemble d'idéaux prônés par le leader et partagés par sa communauté politique. Parmi ces idéaux, nous retrouvons des principes issus de l'éthique des biens communs (cohérents avec la vocation écologiste du mouvement), des valeurs démocratiques défendant une forme de citoyenneté active qui, dans la vision des 5 étoiles, serait encouragée par l'utilisation intensive du réseau Internet de la part de citoyens informés et intéressés par la chose publique. De par leur extranéité aux partis politiques, ces citoyens ordinaires seraient en mesure de défendre l'intérêt collectif et de lutter contre la corruption.

Si le mouvement nous aide à relever le caractère paradoxal des démocraties occidentales, il n'est pas exempte lui-même de contradictions. En effet, la mort des partis ne garantit pas forcement le déclin de la corruption et du fonctionnement autoréférentiel de la politique.

La réduction du mandat électoral à deux législatures n'est pas non plus la seule solution viable pour assurer la défense du bien commun. En effet, le processus de déprofessionnalisation de la politique, déjà en acte au sein des partis traditionnels, pourrait encourager le primat du sens commun sur les savoirs spécialisés et scientifiques, occulter les pensées critiques et encourager ce qu'Alexis de Tocqueville a défini dans les termes d'une "tyrannie de la majorité" (de Tocqueville, 1986).

Même au niveau de l'expérimentation des formes de démocratie directe nous avons identifié des dérives potentielles. Dans le blog, le processus de délibération n'est pas claire, il n'est pas réellement possible de comprendre comment les idées qui émergent de la discussion des internautes sont sélectionnées et contribuent à l'élaboration du programme politique. Nous n'avons pas non plus pu comprendre comment les contenus des sondages proposés dans le blog ont été formulés avant de passer au crible des inscrits. Ce qui fait présumer une persistance de formes de prise de décision verticales qui sont dissimulées derrière une rhétorique de la participation citoyenne.

Figure 1 : Synthèse du discours politique du Mouvement 5 étoiles

\begin{tabular}{|l|l|l|l|l|}
\hline Thèmes & $\begin{array}{l}\text { La politique } \\
\text { traditionnelle }\end{array}$ & $\begin{array}{l}\text { La politique des } 5 \text { é } \\
\text { toiles }\end{array}$ & Idéaux de référence & Points critiques \\
\hline
\end{tabular}




\begin{tabular}{|c|c|c|c|c|}
\hline $\begin{array}{l}\text { Conception } \\
\text { de l'État }\end{array}$ & 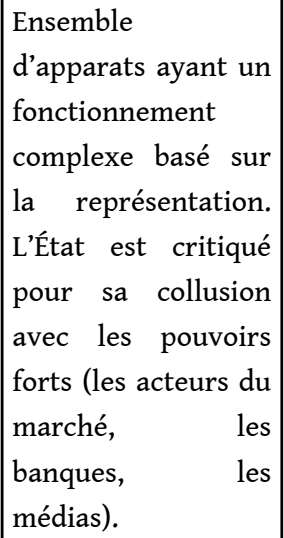 & $\begin{array}{l}\text { L'État doit être } \\
\text { composés par des } \\
\text { citoyens ordinaires, } \\
\text { avec un casier } \\
\text { judiciaire vierge, } \\
\text { qui n'ont autre } \\
\text { prérogative que la } \\
\text { défense du bien } \\
\text { collectif. }\end{array}$ & $\begin{array}{l}\text { Défense de l'intérêt } \\
\text { collectif } \\
\text { Moralité et } \\
\text { intégrité de État. }\end{array}$ & $\begin{array}{lr}\text { Création de lobbies } \\
\text { de } & \text { citoyens } \\
\text { reproduisant } & \text { le } \\
\text { système } & \text { auto- } \\
\text { référentiel } & \text { des } \\
\text { partis politiques. }\end{array}$ \\
\hline $\begin{array}{l}\text { Conception } \\
\text { de la praxis } \\
\text { politique }\end{array}$ & \begin{tabular}{lrr} 
La & \multicolumn{2}{r}{ politique } \\
traditionnelle & est \\
critiquée & pour & sa \\
tendance & à & la \\
corruption, à & la \\
reproduction & des \\
privilèges et & des \\
inégalités. & Elle & est \\
considérée la source \\
principale & de \\
l'aliénation & \\
politique & & des \\
citoyens. &
\end{tabular} & $\begin{array}{l}\text { La politique doit } \\
\text { être conçue comme } \\
\text { une mission civique } \\
\text { temporaire et non } \\
\text { comme un métier. } \\
\text { La limitation des } \\
\text { charges publiques à } \\
\text { deux législatures } \\
\text { garantirait } \\
\text { l'indépendance des } \\
\text { élus ainsi que la } \\
\text { défense des intérêts } \\
\text { collectifs. }\end{array}$ & $\begin{array}{l}\text { Moralisation et dé- } \\
\text { professionnalisation } \\
\text { de la politique }\end{array}$ & $\begin{array}{l}\text { Le sens commun } \\
\text { pourrait } \\
\text { substituer } \\
\text { savoirs } \\
\text { Le fait que le pécialisés. } \\
\text { grand } \\
\text { participe aux prises } \\
\text { de décision ne } \\
\text { garantit pas la } \\
\text { qualité et le bon } \\
\text { fonctionnement du } \\
\text { débat. }\end{array}$ \\
\hline
\end{tabular}

\begin{tabular}{|c|c|c|c|c|}
\hline Thèmes & $\begin{array}{l}\text { La politique } \\
\text { traditionnelle }\end{array}$ & $\begin{array}{l}\text { La politique des } 5 \\
\text { étoiles }\end{array}$ & Idéaux de référence & Points critiques \\
\hline $\begin{array}{l}\text { Conception de } \\
\text { la démocratie }\end{array}$ & $\begin{array}{l}\text { La politique } \\
\text { traditionnelle est ax } \\
\text { ée sur les mé } \\
\text { canismes de la repré } \\
\text { sentation qui é } \\
\text { loignent le citoyen } \\
\text { de la chose publique } \\
\text { et de son exercice. }\end{array}$ & $\begin{array}{l}\text { Les citoyens } \\
\text { devraient } \\
\text { pouvoir accéder } \\
\text { directement aux } \\
\text { charges } \\
\text { publiques et au } \\
\text { processus } \\
\text { décisionnel. }\end{array}$ & $\begin{array}{l}\text { Démocratie directe. } \\
\text { Participation } \\
\text { directe au } \\
\text { processus législatif. }\end{array}$ & $\begin{array}{l}\text { Le recours } \\
\text { constant au vote } \\
\text { numérique } \\
\text { pourrait aplatir } \\
\text { le débat sur les } \\
\text { contenus. }\end{array}$ \\
\hline
\end{tabular}




\begin{tabular}{|c|c|c|c|c|}
\hline $\begin{array}{l}\text { Conception de } \\
\text { la } \\
\text { communication } \\
\text { médiatique }\end{array}$ & $\begin{array}{l}\text { Les médias main } \\
\text { stream sont conçus } \\
\text { comme des outils de } \\
\text { propagande aptes à } \\
\text { promouvoir les idées } \\
\text { dominantes et à dé } \\
\text { fendre les pouvoirs } \\
\text { forts. }\end{array}$ & $\begin{array}{l}\text { Les médias } \\
\text { numériques } \\
\text { encouragent la } \\
\text { libre circulation } \\
\text { de l'information, } \\
\text { la participation } \\
\text { des citoyens au } \\
\text { débat public et } \\
\text { aux processus } \\
\text { décisionnels. }\end{array}$ & $\begin{array}{l}\text { Absence de } \\
\text { médiations. } \\
\text { Transparence dans } \\
\text { la gestion de la } \\
\text { chose publique. } \\
\text { Citoyenneté } \\
\text { numérique active. } \\
\text { Horizontalité }\end{array}$ & $\begin{array}{l}\text { Dictature de la } \\
\text { majorité au sens } \\
\text { de Tocqueville. } \\
\text { Les médias } \\
\text { numériques } \\
\text { n'ont pas un } \\
\text { pouvoir } \\
\text { démocratisant } \\
\text { intrinsèque mais } \\
\text { ils peuvent servir } \\
\text { des intérêts } \\
\text { hégémoniques. }\end{array}$ \\
\hline $\begin{array}{l}\text { Conception de } \\
\text { l'Europe }\end{array}$ & $\begin{array}{l}\text { L'Europe est conçue } \\
\text { comme une instance } \\
\text { autoritaire amenant } \\
\text { les états à d'accepter } \\
\text { les compromis avec } \\
\text { le marché en dépit } \\
\text { des intérêts et des } \\
\text { volontés des } \\
\text { peuples. }\end{array}$ & $\begin{array}{l}\text { L'Europe doit } \\
\text { être } \\
\text { démocratisée } \\
\text { (par } \quad \text { exemple } \\
\text { avec le recours à } \\
\text { des référendums } \\
\text { permettant } \\
\text { peuples }\end{array}$ & $\begin{array}{l}\text { - Justice globale } \\
\text { Défense de la } \\
\text { souveraineté } \\
\text { populaire } \\
\text { Autodétermination } \\
\text { des peuples. }\end{array}$ & $\begin{array}{l}\text { Dérives } \\
\text { nationalistes. }\end{array}$ \\
\hline
\end{tabular}

\section{Conclusion}

52 L'approche cognitiviste nous a permis de réhabiliter le rôle des idées et des représentations sociales et de les réintégrer dans l'étude des phénomènes contestataires. De ces travaux découle donc une conclusion importante: le collectif contestataire, en tant que lieu de construction d'une nouvelle identité politique, revêt une fonction expressive et se définit en vertu des liens sociaux particuliers qui sont tissus à son intérieur.

Ces dynamiques sont partiellement redéfinies par l'interaction avec l'environnement numérique. Dans cette perspective, l'espace info-communicationnel du blog doit être appréhendé à l'intérieur d'une dynamique conflictuelle vis-à-vis de la sphère publique bourgeoise d'Habermas représentant une entité officielle et vouée donc à répéter le langage et la symbolique de l'ordre politique et économique (Negt, 2007). Dans le cadre de ce nouveau militantisme informationnel, l'action contestataire est nourrie par la libre circulation des discours politiques et par la constitution de nouveaux espaces oppositionnels.

54 L'enquête révèle aussi que la désertion des organisations politiques traditionnelles ne semble pas impliquer une disparition de la dimension idéologique. De nouvelles idéologies, souvent liées aux représentations sociales de la technique ou faisant appel à des valeurs démocratiques et libertaires, démontrent leur capacité à forger les usages militants du web (Granjon, 2001; George, 2008) et à nourrir la critique sociale. 
55 Les pratiques encouragées par le réseau Internet sont alors interprétées par les militants comme des actions émancipatrices. C'est à travers ces pratiques que les activistes de Grillo se construisent en opposition avec la pensée dominante, incarnée par les médias et les partis. L'éthique « anti-politique » s'exprime donc par des actes info-communicationnels à travers lesquels le militant déserte l'espace du pouvoir et contribue à construire, en marge, une identité contestataire. Notre étude démontre donc également que le modèle du médium alternatif reste présent sur Internet. Produire, diffuser et rechercher de l'information en dehors des canaux officiels semblent donc être devenues des activités proprement résistantes. Dans le blog de Beppe Grillo, la discussion autour de l'actualité politique a accentué la défiance vis-àvis de l'appareil du parti et la perception d'un vide de représentativité. Face à une crise politique devenue presque chronique, le cadre contestataire du mouvement a été donc progressivement élaboré autour du dépassement $\mathrm{du}$ concept de démocratie représentative.

56 L'ensemble des pratiques qui se développent dans l'espace du blog doit donc être appréhendé comme une réponse contestataire aux limites délibératives qui traversent les démocraties occidentales. Toutefois, si, d'un côté, ces nouveaux espaces publics peuvent attirer des individualités d'horizons différents, d'un autre côté, ils ont aussi tendance à encourager un processus d'individualisation de la politique et de radicalisation des idées plutôt que d'ouverture. Les interventions des militants/usagers constituent alors des tentatives individuelles d'aligner les cadres politiques subjectifs au cadre contestataire du mouvement (Benford et Snow, 2000). Le processus d'alignement des cadres ne fait pas en outre abstraction de logiques verticales et instrumentales. Dans le blog satirique de Grillo, l'éthique de la discussion d'Habermas apparaît en effet soumise à une logique de consommation qui a été visiblement intégrée par les usagers. Cette logique est d'ailleurs aussi activée en amont par le biais de l'attrait suscité par une figure qui, pour le style de ses performances publiques et de ses écrits, reste encore liée au monde du spectacle. La satire et la critique voyagent donc à l'intérieur d'un même espace de confrontation où le blogueur oriente et écoute à la fois ses partisans.

Malgré les nombreux points problématiques que nous avons soulevé, ce mouvement constitue un moteur de renouvellement du système politique national. Son intégration dans les institutions italiennes a en effet favorisé la dialectique, affaibli la logique de l'alternance et introduit une forme de démocratisation des accès à la sphère politique.

Le développement de pratiques délibératives propres et de formes d'auto-organisation politique décide en effet du dépassement du stade oppositionnel et peut impliquer un processus d'intégration de ces espaces alternatifs dans le jeu de la représentation institutionnelle (Negt, 2007, p. 19). Dans ce nouveau contexte du militantisme, les intérêts de classe sont remplacés par un combat pour l'affirmation du droit à communiquer, à s'informer d'une manière alternative (Cardon et Granjon, 2010) et à participer au débat démocratique en ligne (George, 2008).

59 Mais si les activistes réclament leur droit à la communication, les logiques du marché global tendent à la transformer en un ensemble de services circulant, d'une manière asymétrique, dans le libre marché des idées (Mattelart, 1999). C'est à partir de ce constat qu'une perspective critique dans l'étude de la communication se rend nécessaire afin d'envisager une fonction démocratique de la technologie dans notre société. 


\section{BIBLIOGRAPHIE}

Bibliographie

Zygmunt Bauman, La vie Liquide, Arthème Fayard/Pluriel, Paris, 2013.

Robert D. Benford et David A. Snow, « Framing processes and social movements : an overview and assessment ", Annual Review of Sociology, vol. 26, 2000, p. 611-639.

Robert D. Benford et David A. Snow, « Master frames and cycles of protest », in A. D. Morris et C. McClurg Mueller, (dir.), Frontiers in social movement theory, University of Yale Press, New Haven, 1992, p.133-155.

Dominique Cardon,. « La participation en ligne », Idées économiques et sociales, vol. 3 (173), 2013, p. 33-42.

Dominique Cardon et Fabien Granjon, Médiactivistes, Presse de Science Po, Paris, 2010.

Manuel Castells, Communication Power, Oxford University Press, Oxford, 2009.

Piergiorgio Corbetta et Elisabetta Gualmini (dir), Il partito di Grillo, Il Mulino, Bologna, 2013.

Peter Dahlgren, Media and Political engagement, Citizens, communication and democracy, Cambridge University Press, Cambridge, 2009.

Patrice Flichy, « Internet, un outil de la démocratie? », La Vie des idées, 14 janvier 2008.

Éric George, « De la complexité des relations entre démocratie et TIC », Nouvelles pratiques sociales, vol. 21 (1), 2008, p. 38-51.

Éric George, « Les usages militants d'Internet », Communication, vol. 22 (2) , 2003, p. 99-124.

Fabien Granjon, L'Internet militant, Mouvement social et usages des réseaux télématiques, Editions Apogée, Rennes, 2001.

Jürgen Habermas, Théorie de l'agir communicationnel, Tome 2, Pour une critique de la raison fonctionnaliste, Fayard, Paris, 1987.

Jacques Ion, Spyros Franguiadakis et Pascal Viot, Militer aujourd'hui, Éditions Autrement, Paris, 2005.

Romain Lecomte, «Internet et la reconfiguration de l'espace public tunisien : le rôle de la diaspora ", tic\&société, vol. 3 (1-2), 2009, p. 198-229.

Lilian Mathieu, La démocratie protestataire : mouvements sociaux et politique en France aujourd'hui, Presses de Science Po, Paris, 2011.

Armand Mattelart, La communication-monde, histoire des idées et des stratégies, La Découverte, Paris, 1999.

Bernard Miège, Espace Public Contemporain, PUG, Grenoble, 2010.

Laurence Monnoyer-Smith, Communication et délibération. Enjeux technologiques et mutations citoyennes, Lavoisier/Hermès science, Paris, 2011.

Vincent Mosco, The Digital Sublime, MIT Press, MA, 2004.

Pierre Musso, Réseaux et société, PUF, Paris, 2003. 
Oskar Negt, L'espace public oppositionnel, L'expérience plébéienne, une histoire discontinue de la liberté politique, Payot, Paris, 2007.

Hartmut Rosa, Aliénation et accélération, vers une théorie critique de la modernité tardive, La

Découverte, Paris, 2012.

Pierre Rosanvallon, La contre-démocratie, la politique à l'âge de la défiance, Seuil, Paris, 2006.

Alexis De. Tocqueville, De la démocratie en Amérique, Robert Laffont, Paris, 1986.

\section{NOTES}

1. Le "master frame" constitue un cadre contestataire général qui peut être épousé par différents mouvements dont les revendications sont rassemblées à l'intérieur d'un même cycle protestataire. C'est le cas des mouvements altermondialistes avec lesquels le mouvement de Grillo partage la critique du marché financier global et le primat de l'économie sur le politique.

2. Parmi ces travaux, il émerge l'analyse de Zuo et Benford sur la mobilisation étudiante de Pekin en 1989. Les deux auteurs constatent que les étudiants se sont appropriés de l'idéologie dominante pour la détourner contre le pouvoir et réduire la possibilité d'activation d'un contrecadrage de la part des autorités (Zuo, Benfort, 1995). Cette étude montre clairement que dans le cas où les groupes contestataires visent à modifier significativement l'ordre social, ils doivent se confronter automatiquement avec l'idéologie dominante qui assure la reproduction matérielle et idéologique du système en place.

3. Nous pouvons par exemple présumer que les groupes luttant pour les droits des homosexuels se placent à contre courant par rapport à l'appareil idéologique familial. De la même façon, les médiactivistes (Cardon et Granjon, 2010) sont engagés dans des tentatives de déstructuration de l'appareil médiatique dominant.

4. Le concept de populisme, souvent utilisé avec une acception négative pour décrire des stratégies rhétoriques de manipulation qui font appel à la souveraineté du peuple, constitue en réalité une catégorie sémantique plutôt fluide. Elle renferme en effet des éléments contradictoires qui vont de l'autoritarisme à la promotion de formes de démocratie directe. Le recours à des techniques de communication ou à des catégories idéologiques "populistes" concerne tant les partis traditionnels, qui essaient de trouver des nouvelles formes de légitimation, que les mouvements sociaux, souvent faisant recours à la dimension anti-système et anti-élitiste du populisme.

5. La coopérative ouvrière, la cité ouvrière ou même le syndicat, en agrégeant des intérêts et des expériences réelles, constituent des exemples d'espaces publics prolétariens (Negt, 2007, p. 100). Il s'agit d"'espaces publics partiels" qui représentent la sphère de la production tout en contribuant également au processus de politisation des travailleurs.

6. Au début des années 2000 , nous avons assisté à l'explosion du phénomène des télés de rue, en 2009, Le Peuple des violets, un groupe créé sur Facebook, a été à l'origine du NoBerlusconiDay. En juin 2011, la blogosphère et les collectifs citoyens en ligne ont eu un poids déterminant sur la victoire du «Oui » lors d'un référendum qui a sanctionné les projets de loi du gouvernement Berlusconi.

7. Lors des élections législatives de février 2013, le Mouvement 5 étoiles a obtenu le plus grand nombre de votes $(25,5 \%)$ acquis individuellement par un parti à la Chambre des députés. Le mouvement constitue actuellement la principale force d'opposition au Parlement italien.

8. La situation délicate ressortie des résultats des urnes a amené le Président de la République Giorgio Napolitano à nommer Enrico Letta (du Parti Démocrate) à la tête d'un gouvernement d'union nationale. Suite à la démission de ce dernier, Matteo Renzi, le néo-secrétaire du Parti 
Démocrate, a récemment été chargé de former un deuxième gouvernement soutenu par le centre-gauche et par le Nouveau Centre-droit d'Angelino Alfano. L'entrée du Mouvement 5 étoiles dans la sphère politique italienne a ainsi provoqué un basculement du système représentatif, d'un bipolarisme traditionnel entre gauche et droite vers une nouvelle configuration politique autour de trois forces principales.

9. Extrait du non-statut du Mouvement 5 étoiles consultable à la page https://s3-euwest-1.amazonaws.com/materiali-bg/Regolamento-Movimento-5-Stelle.pdf

10. Les communiqués politiques peuvent être consultés à la page http://www.beppegrillo.it/ movimento/comunicati-politici.html de l'espace du blog dédié au mouvement.

11. Le Medef italien.

12. Les concepts de « servitude ", $d^{\prime}$ " esclavage » et d' « asservissement » sont souvent employés dans le blog.

13. Dans les communiqués, nous retrouvons en effet de nombreuses expressions renvoyant à la dimension individuelle de l'action politique : «chaque italien doit se mettre en jeu », « le citoyen est le seul responsable de son destin ", " donner aux citoyens la responsabilité de leurs choix ».

14. En novembre 2009, dans le communiqué politique numéro 28 , Grillo déclare que "la politique est morte, depuis long temps". Le communiqué est consultable à la page http:// www.beppegrillo.it/2009/11/comunicato_poli_19.html (dernière consultation 19/09/2014). Mais la mort de la politique renvoie aussi aux trois mythes composant l'imaginaire du cyber-espace (la fin de la politique, de l'histoire et de la géographie) ainsi qu'ils ont été identifiés par Vincent Mosco dans "The digital sublime" (Mosco, 2004).

\section{RÉSUMÉS}

Notre travail vise à identifier le rôle revêtu par les pratiques discursives qui animent la blogosphère politique dans la construction des nouveaux "cadres contestataires". Fondée sur les méthodes de l'observation et de l'analyse de contenu et portant sur le cas italien du Mouvement 5 étoiles, l'enquête montre comment le dispositif du blog du comique italien Beppe Grillo contribue à définir le processus de cadrage et, d'une manière plus générale, à façonner la conscience protestataire $\mathrm{du}$ mouvement. Conçu en tant qu'espace anti-hégémonique et oppositionnel, le blog fonctionne à la fois comme un producteur de contre-information et comme un outil puissant de mobilisation et d'action politique.

Our work aims to identify the role played by the discursive practices that are hosted in the political blogosphere in the construction of the new "collective frames". Based on the observation and content analysis methods and focused on the Italian case of the Five stars movement, this inquiry shows how the apparatus of the Italian comedian Beppe Grillo's blog contributes to define the framing processes and, in a general way, to shape the protesting conscience of the movement. Conceived as a counter-hegemonic and oppositional space, the blog works at the same time as a provider of counter-information and as a powerful mean of mobilisation and political action. 
INDEX

Mots-clés : Mots-clés : pratiques numériques ; mobilisation sociale; espace public oppositionnel; cyber-activisme ; mouvements sociaux ; cadres de l'action collective.

Keywords : Keywords : digital practices; social mobilisation; oppositional public sphere; cyberactivism; social movements; collective frames.

\section{AUTEUR}

\section{PAOLA SEDDA}

Maître de conférences en SIC à l'Université de Bourgogne

Laboratoire CIMEOS 